Background: Hypovolemia and fluid imbalance are quite common after aneurysmal SAH and negatively affect the clinical outcome. Hypovolemia correlates with the clinical grade of SAH and may exacerbate vasospasm. Goal-directed fluid therapy (GDFT) utilizes cardiac output (CO) monitoring techniques to guide clinicians for administering fluids, vasopressors, and inotropes. We compared hemodynamic effects and postoperative outcome of patients receiving intraoperative GDFT using left ventricular outflow tract (LVOT) velocity time integral (VTI) by transesophageal echocardiography (TEE) with the CVP-guided conventional fluid therapy in patients undergoing clipping of aneurysm.

Materials and Methods: Fifty adult ASA I and II patients with subarachnoid hemorrhage secondary to aneurysm rupture, scheduled to undergo clipping surgery were included. In the preoperative period, transthoracic echocardiography (TTE) of all patients was done to rule out stunned myocardium or valvular abnormalities. The study patients were then randomly divided into two groups; Group V $(n=25)$, patients received GDFT using LVOT-VTI. LVOT-VTI was measured with TEE in transgastric long-axis view and kept between 15 and $25 \mathrm{~cm}$. Group C ( $n=25)$, Patients received CVP-guided fluid therapy and a CVP of 8 to $10 \mathrm{~cm}$ of water was aimed intraoperatively.

Results: Both the groups had similar heart rates and mean blood pressures intraoperatively throughout the duration of surgery. However, intraoperative fluid administration to Group V was significantly less than that administered to group $C(p<0.0001)$. The baseline serum lactate levels as well as the lactate levels at the end of surgery were comparable in the two groups. The average intraoperative urine output, blood loss, and the average amount of blood administered were also similar in the two groups. Postoperatively, both the groups had similar durations of postoperative mechanical ventilation and ICU stay. The incidence of postoperative complications like ventilator-associated pneumonia, sepsis, vasospasm, and in-hospital mortality were also comparable. MRS scores at discharge $(p=0.169)$ and eGOS at $1(p=0.48)$ and 3 months ( $p=0.556$ ) were comparable in the two groups.

Conclusions: Intraoperative GDFT using TEE in aneurysmal clipping is viable and results in lesser amount of intraoperative fluid administration while maintaining stable hemodynamics during surgery.

\section{A006 Anesthetic Challenges of an Infant with Apert Syndrome and Tetralogy of Fallot for Craniosynostosis Correction \\ Satish K. Sundararajan, ${ }^{1}$ Karen R. Lionel, ${ }^{1}$ Ramamani Mariappan ${ }^{1}$ ${ }^{1}$ Department of Anaesthesia, Christian Medical College, Vellore, India}

Background: Apert syndrome (ApS) is characterized by craniosynostosis, craniofacial anomalies, and symmetrical syndactyly. Approximately $10 \%$ of Apert syndrome children can have associated congenital cardiac anomalies. It is rare to see ApS with tetralogy of Fallot (TOF). Craniosynostosis corrective surgery is associated with massive bleeding and venous air embolism. The presence of TOF increases the risks of perioperative morbidity and mortality.

Case Description: An 8-month-old infant (weight $6 \mathrm{~kg}$ ), diagnosed with ApS and TOF was planned for a bifrontal craniotomy with frontal advancement and right frontal orbitotomy. As the child had difficult venous access, it was decided to perform gas induction. After standard monitoring, induction was performed with sevoflurane (4\%). An intravenous (IV) line was secured quickly, anesthesia was deepened with fentanyl $(1.5 \mu \mathrm{g} / \mathrm{kg})$, ketamine $(1.0 \mathrm{mg} / \mathrm{kg})$, and propofol ( $1 \mathrm{mg} / \mathrm{kg}$ ) and sevoflurane concentration was decreased (2\%). Intubation was performed using a video laryngoscope. Arterial and the subclavian triple lumen was established. Scalp block, fentanyl, and morphine were given for analgesia. A loading dose of tranexamic acid (20 mg/kg), followed by an infusion ( $1 \mathrm{mg} / \mathrm{kg} / \mathrm{h}$ ) was given to reduce the bleeding. Scalp dissection and the strip cranioplasty resulted in 150 to $170 \mathrm{~mL}$ of blood loss, which was replaced with packed red blood cells (100 $\mathrm{mL})$, fresh frozen plasma $(50 \mathrm{~mL})$, and cryoprecipitate $(25 \mathrm{~mL})$. A low dose of noradrenaline was started $(0.02-0.05 \mu \mathrm{g} / \mathrm{kg} / \mathrm{min})$ to maintain the systemic vascular resistance (SVR). The child remained warm, hemodynamically stable, and the $\mathrm{SpO}_{2}$ was varying between 85 and 93\%. At the end of surgery, the child was extubated and shifted to the neurosurgical ICU. The main anesthetic challenges were the management of difficult airway, C-MAC video laryngoscope helped us to secure the airway. Maintaining the SVR using noradrenaline and avoiding factors which can increase the pulmonary vascular resistance prevented intraoperative "Tet spell." Transfusion of blood and blood products helped maintaining the hemodynamic stability.

Conclusions: Meticulous planning and administration of titrated anesthetics to maintain both cardiovascular and the cerebrovascular homeostasis is paramount important for a successful outcome.

A007 An Unusual Presentation of Guillain-Barré Syndrome with Cervicobulbar Palsy Ujjwal Daspal, ${ }^{1}$ Rekha Gupta, ${ }^{1}$ Mudit Garg, ${ }^{1}$ Deepak Goel ${ }^{2}$

${ }^{1}$ Department of Anaesthesia, Max Institute of Neurosciences, Dehradun, Uttarakhand, India

2Department of Neurology, Max Institute of Neurosciences, Dehradun, Uttarakhand, India

Background: Guillain-Barré syndrome (GBS) is an autoimmune disorder. Flaccid paralysis is the hallmark of this disease, there are some rare variants which may be easily missed unless suspected. Here, we present a case of rare pharyngeal-cervical-brachial (PCB) variant of GBS with associated lower motor neuron type of facial palsy. The PCB variant is defined by rapidly progressive oropharyngeal and cervicobrachial weakness, and is often misdiagnosed as brainstem stroke, myasthenia gravis, or botulism.

Case Description: A 48-year-old female patient presented to us with complaints of breathing difficulty, which increased markedly on the same day, followed by facial 